\title{
Imagination and remembrance: what role should historical epidemiology play in a world bewitched by mathematical modelling of COVID-19 and other epidemics?
}

\author{
George S. Heriot ${ }^{1,4}{ }^{\oplus} \cdot$ Euzebiusz Jamrozik $^{2,3,4}$
}

Received: 12 October 2020 / Accepted: 21 April 2021 / Published online: 7 June 2021

(C) Springer Nature Switzerland AG 2021

\begin{abstract}
Although every emerging infectious disease occurs in a unique context, the behaviour of previous pandemics offers an insight into the medium- and long-term outcomes of the current threat. Where an informative historical analogue exists, epidemiologists and policymakers should consider how the insights of the past can inform current forecasts and responses.
\end{abstract}

Keywords COVID $19 \cdot$ Epidemiology $\cdot$ Epidemiologic methods $\cdot$ History/ epidemiolgy $\cdot$ Models $\cdot$ Statistical

\section{Text}

The emergence of COVID-19 has seen an explosion of epidemiological models seeking to characterise and forecast the course of the pandemic. The outputs of these models have influenced policy decisions around the world despite extremely uneven forecasting performance of similar models of other recent emerging infectious

Topical Collection "Seeing Clearly Through COVID-19: Current and future questions for the history and philosophy of the life sciences", edited by G. Boniolo and L. Onaga.

George S. Heriot

george.heriot@mh.org.au

1 School of Public Health and Preventive Medicine, Monash University, 553 St Kilda Road, Melbourne VIC 3004, Clayton, VIC, Australia

2 The Ethox Centre \& Wellcome Centre for Ethics and the Humanities, Nuffield Department of Population Health, University of Oxford, Oxford, UK

3 Monash Bioethics Centre, Monash University, Clayton, VIC, Australia

4 Royal Melbourne Hospital Department of Medicine, University of Melbourne, Parkville, VIC, Australia 
diseases. Instead, one might look to data from past pandemics to inform current risk assessments. Some view such analogies to events of the past as unreliable, raising the reductionist truism that every combination of disease and context is unique (Peckham 2020). However, both epidemiological modelling of future scenarios and analyses of historical data are liable to errors of inputs, assumptions and interpretations; in this paper we argue that both techniques should be considered "wrong, but useful" (Christley et al. 2013) and that greater awareness of historical data may improve pandemic preparedness and responses.

The construction of an epidemiological model incorporates structural assumptions about the system under study and requires the assembly of input data describing the specific context and disease. Complex biological systems resist this simple parametrisation and models of these systems necessarily involve simplifications whose impact on the predictive skill of the model are difficult to quantify. In the early phase of a new epidemic, the input conditions for these models are gleaned from imperfect observations affected by, for example, ascertainment, time-based, and reporting biases that undermine both their accuracy and precision. The sensitivity of these models to their input conditions, and the appropriateness and stability of their structural assumptions, lends substantial uncertainty to any predictions made; their extrapolation beyond the initial time, place, or pathogen is even less secure.

In contrast to mathematical models, the use of historical data for forecasting contemporary epidemics does not require simplifying assumptions as to the mechanisms of epidemic propagation or the sociogeographical structure of affected communities. Instead, this approach relies on the comparability of the current disease and context to previous diseases and contexts, similar to the analogue method used for weather forecasting prior to the availability of sufficient reliable data and computing power. On the one hand, many features of the COVID-19 pandemic are inextricably linked to contemporary circumstances and particular contexts. Local experiences of epidemics among citizens, patients and clinicians will therefore vary. On the other hand, although medical science has advanced considerably in recent centuries, the spread of respiratory viruses between human hosts has changed little for millennia. People are infected in the same way, suffer in the same way, and die in the same way. Therefore, with respect to the transmission and sequelae of pandemic viruses, twenty-first century human communities may bear greater resemblance to communities in the eighteenth and nineteenth centuries than to an abstracted representation within an epidemiological model. Moreover, epidemiological studies of the variation of the expression of past pandemics in different communities may be more informative for current pandemic responses than model simulations based on combinations of uncertain abstract input variables.

Once a new pandemic appears to fit in the range of those observed before, the behaviour and impact of previous pandemics should be considered rather than discarded. Consultation of historical data reveals the significant similarities between the respiratory viral pandemics of the last few centuries in general (Patterson 1986; Valleron et al. 2010) and also the availability of reasonable analogues for the specific 
epidemiological observations of COVID-19. The infectivity and severity of SARSCoV-2, whether assessed by statistical parameterisation (basic reproduction number ${ }^{1}$ and adjusted case or infection fatality ratios, ${ }^{2}$ respectively) or synoptic description (household attack rate, ${ }^{3}$ time to epidemic peak, ${ }^{4}$ and excess all-cause mortality rates $^{5}$ ), are well within the range described by respiratory viral pandemics of the last few centuries (where the 1918-20 influenza is the clear outlier). The variation in estimates for these parameters as they apply to COVID-19 is no narrower than those calculated from historical observations made at different locations during previous pandemics.

Perhaps the best available historical analogue for COVID-19 is the 1889-91 pandemic of "la Grippe," attributed either to an H3N8 influenza virus(Dowdle 1999) or to the emergence of human coronavirus OC43 - now a globally endemic "common cold" virus (Vijgen et al. 2005). This late nineteenth century pandemic has compelling similarities with our current experience, both superficial (including the early illness of a British prime minister, febrile media coverage, prominence of post-infectious fatigue syndromes, and xenophobic or conspiratorial origin theories) and with regard to its apparent epidemiological parameters.

Specific epidemiological correlates between the 1889-91 and 2020-21 pandemics include the low morbidity among children, the lack of the shift in excess mortality to younger age groups usually seen with pandemic influenza, the magnitude and distribution of peak excess mortality ratios in metropolitan settings, and the rapidity of epidemic propagation within communities (Valleron et al. 2010; Campbell A. and Morgan E. 2020; Nicoll et al. 2012; Nguyen-Van-Tam et al. 2003; Honigsbaum 2010; Smith 1995). While downscaling this synoptic analogy to make short-term forecasts of COVID-19 activity in any given place 130 years later is clearly foolish (short-range forecasts from well-observed local data being very much the preserve of computational modelling), the historical record may provide a richer and more useful understanding of the range of medium- and long-term consequences of a pandemic of this epidemiological pattern on human societies than even the most complex mathematical model.

Analogies to past pandemics can also provide an important check on the assumptions made during model construction. As an example, every established respiratory pandemic of the last 130 years has caused seasonal waves of infection and has culminated in viral endemicity. Despite this robust observation, initial models of COVID19 structurally excluded this possibility through the failure to incorporate seasonal transmission effects, or either pre-existing or partial post-infection immunity to infection. Although SARS-CoV-2 is a novel non-influenza pathogen, the strong

\footnotetext{
${ }^{1}$ The number of new infections generated by each infectious individual in a given context assuming a fully-susceptible population.

${ }^{2}$ The proportion of (identified) individuals who die from infection, often adjusted for age or other factors.

3 The proportion of household contacts who contract infection from an index case.

${ }^{4}$ The time between the first detected case and the highest daily incidence of infection in a population.

${ }^{5}$ The difference in the total number of deaths during a pandemic as compared to a previous comparable period.
} 
seasonal behaviour of closely-related endemic coronaviruses seems a more reliable starting point than the assumption of an unprecedented weather-agnostic respiratory pathogen causing permanent sterilising natural immunity. Recent COVID-19 models incorporating these minimal additional complications demonstrate the resulting deterministic chaos, highlighting both the limitations of current mathematical approaches and the need to consider other sources of guidance for anything more than short-term forecasts (Dalziel et al. 2016; Saad-Roy et al. 2020). Model extrapolations suggesting that COVID-19 will have consequences out of proportion to other comparable respiratory pandemics should be viewed with suspicion rather than as a sound counterfactual used to justify aspects of the pandemic response.

While some degree of epistemic humility (Jones 2020) is prudent, the apparent bias in favour of modelling techniques over analyses of historical data should be discarded. Rather than relying only on mathematical models of the future, researchers and policymakers should consider how knowledge of the past might assist in understanding the likely consequences of COVID-19 and future respiratory viral pandemics.

\section{References}

Campbell A. \& Morgan E. 2020. Comparisons of all-cause mortality between European countries and regions: January to June 2020. ed. O. f. N. Statistics. United Kingdom.

Christley, R. M., M. Mort, B. Wynne, J. M. Wastling, A. L. Heathwaite, R. Pickup, Z. Austin \& S. M. Latham (2013) "Wrong, but useful": negotiating uncertainty in infectious disease modelling. PLoS One, 8, e76277.

Dalziel, B. D., O. N. Bjørnstad, W. G. van Panhuis, D. S. Burke, C. J. Metcalf \& B. T. Grenfell (2016) Persistent chaos of measles epidemics in the prevaccination United States caused by a small change in seasonal transmission patterns. PLoS Comput Biol, 12, e1004655.

Dowdle, W. (1999). Influenza A virus recycling revisited. Bulletin of the World Health Organization, 77, 820

Honigsbaum, M. (2010). The great dread: Cultural and psychological impacts and responses to the Russian'influenza in the United Kingdom, 1889-1893. Social History of Medicine, 23(2), 299-319

Jones, D. S. (2020). History in a crisis - Lessons for Covid-19. New England Journal of Medicine, 382, $1681-1683$

Nguyen-Van-Tam, J. S., \& Hampson, A. W. (2003). The epidemiology and clinical impact of pandemic influenza. Vaccine, 21, 1762-1768

Nicoll, A., Ciancio, B., Chavarrias, V. L., Mølbak, K., Pebody, R., Pedzinski, B., Penttinen, P., van der Sande, M., Snacken, R., \& Van Kerkhove, M. (2012). Influenza-related deaths-available methods for estimating numbers and detecting patterns for seasonal and pandemic influenza in Europe. Eurosurveillance, 17, 20162

Patterson, K. D. 1986. Pandemic influenza, 1700-1900: a study in historical epidemiology. Rowman \& Littlefield Pub Incorporated.

Peckham, R. (2020). COVID-19 and the anti-lessons of history. Lancet, 395, 850-852

Saad-Roy, C. M., Wagner, C. E., Baker, R. E., Morris, S. E., Farrar, J., Graham, A. L., Levin, S. A., Mina, M. J., Metcalf, C. J. E., \& Grenfell, B. T. (2020). Immune life history, vaccination and the dynamics of SARS-CoV-2 over the next 5 years. Science, 370, 811-818.

Smith, F. B. (1995). The Russian influenza in the United Kingdom, 1889-1894. Social History of Medicine, $8,55-73$

Valleron, A. J., Cori, A., Valtat, S., Meurisse, S., Carrat, F., \& Boëlle, P. Y. (2010). Transmissibility and geographic spread of the 1889 influenza pandemic. Proceedings of the National Academy of Sciences of the USA, 107, 8778-8781 
Vijgen, L., Keyaerts, E., Moës, E., Thoelen, I., Wollants, E., Lemey, P., Vandamme, A.-M., \& Van Ranst, M. (2005). Complete genomic sequence of human coronavirus OC43: Molecular clock analysis suggests a relatively recent zoonotic coronavirus transmission event. Journal of Virology, 79, 1595-1604

Publisher's Note Springer Nature remains neutral with regard to jurisdictional claims in published maps and institutional affiliations. 